\title{
To fix or not fix biofilms to study microbial soil aggregation
}

Yuchen Zhang ${ }^{1}$, Jiyoung Son ${ }^{2}$, Qiaoyun Huang ${ }^{3}$, Wenli Chen ${ }^{4}$ and Xiao-Ying Yu ${ }^{2}$

${ }^{1}$ Pacific Northwest National Laboratory, Richland, Washington, United States, ${ }^{2}$ Pacific North West National Laboratory, United States, ${ }^{3}$ Huazhong Agricultural University, Wuhan, China (People's Republic), ${ }^{4}$ Huazhong Agricultural University, China (People's Republic)

Bacterial biofilms are considered to be a main player in organic processing and soil aggregation. Therefore, characterization and understanding of the biofilm interactions with soil components is important in deepening our knowledge in the biosphere and rhizosphere. This work presents two approaches to prepare the bacterial biofilm samples suitable for high resolution mass spectral imaging using time-of-flight secondary ion mass spectrometry (ToF-SIMS). Compared to other mass spectrometry techniques, ToF-SIMS is less destructive, and it offers submicrometer spatial mapping of molecular species of importance in metabolic processes [1]. Shewanella MR-1 was used as the model bacteria biofilm due to their known traits in soil chemistry and microbiology [2]. A mixture of silica, alumina, and iron oxide was used as the model soil system [3].

In the first type of sample preparation, we took a static approach as depicted Fig. 1a. The bacteria were inoculated in a multi-well cell culture dish at their log phase. Then soil components were added to the culturing well. The mixture of the bacteria biofilms and soil components were scratched off carefully using a pipette tip and deposited onto the clean silicon ( $\mathrm{Si}$ ) wafers before ToF-SIMS analysis. In the second approach, we used a microfluidic cell to culture biofilms as depicted Fig. 1b [1, 2]. We made a modification to include a clean Si wafer as the main substrate for biofilm attachment in the microfluidic chamber. The soil component was mixed to the growth media (TSB without dextrose) at a ratio of 1:1 by volume and used as nutrients to support the biofilm's growth after the biofilms reached the log phase in the growth chamber. Samples were collected periodically. In the first static culturing method, a series of $\mathrm{Si}$ wafers were used to capture the temporal progression of the biofilms and the soil components in a course of several days. In the second dynamic method, the effluents were collected onto clean $\mathrm{Si}$ substrates accordingly. The integrated time intervals were chosen based on the growth curve of the strain. An IONTOF GmbH TOF-SIMS V spectrometer was used with a $25 \mathrm{keV} \mathrm{Bi3}{ }^{+}$primary ion beam. The spectral analysis was performed by rastering over a $500 \mu \mathrm{m}$ by $500 \mu \mathrm{m}$ area with a beam current of $\sim 0.32$ pA. On average, 60 to 100 scans were collected.

Figure 2 show the ToF-SIMS spectral comparison of the static and dynamic culturing of Shewanella to study its interaction with the mineral mixture as the soil simulant in the negative ion mode. Distinctive characteristic fatty acids peaks of Shewanella biofilms such as myristic acid $\left(m / z^{-} 227, \mathrm{C}_{14} \mathrm{H}_{27} \mathrm{O}_{2}^{-}\right)$, palmitic acid $\left(m / z^{-} 227, \mathrm{C}_{14} \mathrm{H}_{27} \mathrm{O}_{2}{ }^{-}\right)$, and arachidic acid $\left(\mathrm{m} / \mathrm{z}^{-} 227, \mathrm{C}_{14} \mathrm{H}_{27} \mathrm{O}_{2}{ }^{-}\right)$as well as an interesting biomarker riboflavin peak $\left(\mathrm{m} / \mathrm{z}^{-} 241, \mathrm{C}_{12} \mathrm{H}_{9} \mathrm{~N}_{4} \mathrm{O}_{2}{ }^{-}\right)$are observed in the dynamic setup results. In contrast, the static setup does not seem to provide as much information, indicating that the static approach is not optimal for studying the biofilms and the minerals using ToF-SIMS. Our initial results demonstrate that sample preparation is quite critical in microanalysis of bacteria biofilms, specifically in surface analysis like ToF-SIMS applications. The microfluidic growth chamber seems to be more flexible in microbial culture and media tuning, both are important in simulating a variety of conditions to understand microbes and soil interactions at the microscale. Additionally, characteristic signals of biofilms are not 
buried under the mineral components in the dynamic setup, which is imperative in understanding the role of biofilms in soil aggregation that occurs at the microbe-mineral interface [4].
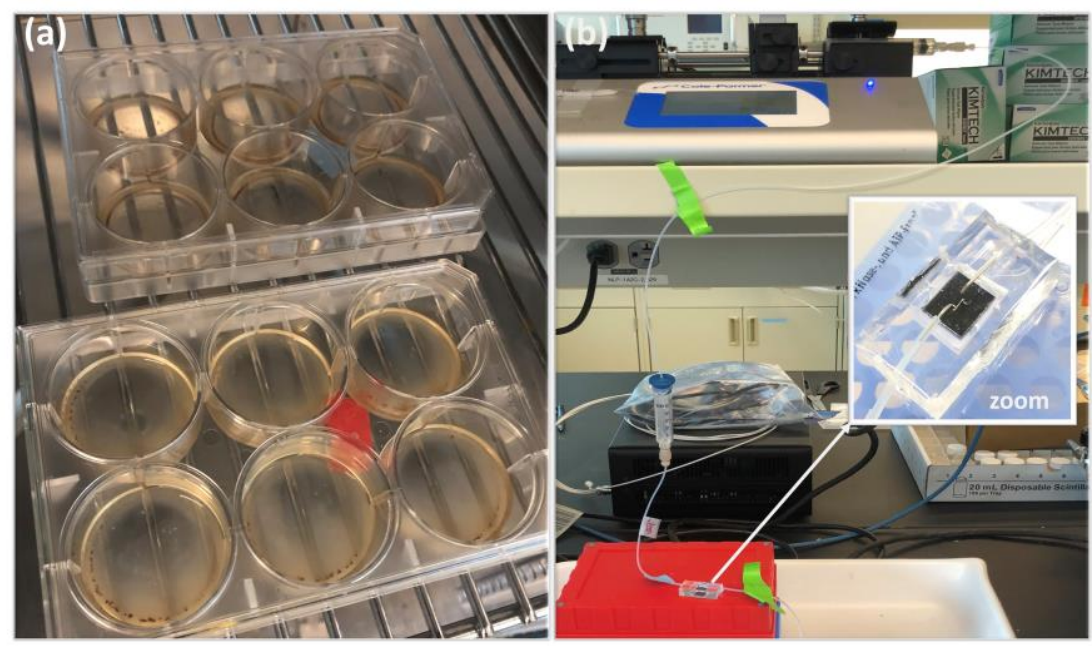

Figure 1. (a) The static and (b) the dynamic culture setup to study the interactions of minerals and biofilms.

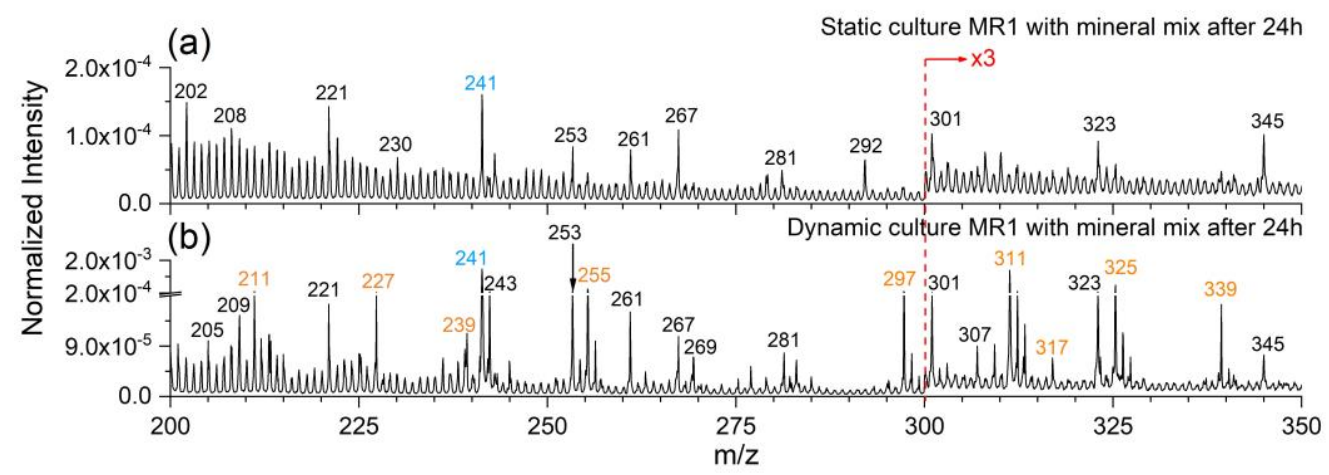

Figure 2. ToF-SIMS negative mode spectral comparison of Shewanella MR-1 interacting with a mineral mixture acquired from the static (a) and dynamic (b) setup, respectively. Fatty acid peaks in orange and riboflavin peak in blue are marked for ease of viewing.

\section{References}

[1] W Wei et al., Molecular insights into communications of Geobacter syntrophic communities, Anal. Chem. (2020), 92 (15), 10402-10411.

[2] Y Ding et al., Molecular evidence of toxic chemicals' impact on the biofilm and its matrix, Analyst (2019), 144, 2498-2503.

[3] Kaze R C et al., Microstructure and engineering properties of $\mathrm{Fe}_{2} \mathrm{O}_{3}(\mathrm{FeO})-\mathrm{Al}_{2} \mathrm{O}_{3}-\mathrm{SiO}_{2}$ based geopolymer composites. Journal of Cleaner Production (2018), 199, 849-859.

[4] We acknowledge support from the PNNL Laboratory Directed Research and Development fund and Yuchen Zhang thanks the Chinese Scholar Council Fellowship for support. 